Case Report

\title{
Transcranial Magnetic Stimulation for the Treatment of Pharmacoresistant Nondelusional Auditory Verbal Hallucinations in Dementia
}

\author{
Anna Marras ${ }^{1,2}$ and Stefano Pallanti ${ }^{3,4}$ \\ ${ }^{1}$ University of Florence, 50137 Florence, Italy \\ ${ }^{2}$ Institute of Neuroscience, 50121 Florence, Italy \\ ${ }^{3}$ University of Florence School of Medicine, 50134 Florence, Italy \\ ${ }^{4}$ Albert Einstein College of Medicine and Montefiore Medical Center, NY 10461, USA \\ Correspondence should be addressed to Stefano Pallanti; stefanopallanti@yahoo.it
}

Received 2 August 2013; Accepted 15 September 2013

Academic Editors: I. G. Anghelescu, J. S. Brar, M. Kluge, and F. Oyebode

Copyright ( 2013 A. Marras and S. Pallanti. This is an open access article distributed under the Creative Commons Attribution License, which permits unrestricted use, distribution, and reproduction in any medium, provided the original work is properly cited.

\begin{abstract}
Auditory verbal hallucinations (AVHs) are known as a core symptom of schizophrenia, but also occur in a number of other conditions, not least in neurodegenerative disorders such as dementia. In the last decades, Transcranial Magnetic Stimulation (TMS) emerged as a valuable therapeutic approach towards several neurological and psychiatric diseases, including AVHs. Herein we report a case of a seventy-six-years-old woman with vascular-degenerative brain disease, complaining of threatening AVHs. The patient was treated with a high-frequency temporoparietal (T3P3) rTMS protocol for fifteen days. A considerable reduction of AVHs in frequency and content (no more threatening) was observed. Although further research is needed, this seems an encouraging result.
\end{abstract}

\section{Introduction}

Transcranial Magnetic Stimulation (TMS) is a noninvasive technique of neuromodulation and neurostimulation based on the principle of electromagnetic induction of an electric field in the brain. It was originally developed for the of study brain function [1]. Since Barker and colleagues first demonstrated TMS, it has been widely used earlier to investigate motor conduction and control, later in the study of cognitive functions and brain plasticity. TMS is widely studied in the diagnosis and treatment of neurological and psychiatric diseases, too.

A hallucination is often referred to as "a sensory experience, which occurs in the absence of corresponding external stimulation of the relevant sensory organ, has a sufficient sense of reality to resemble a veridical perception, over which the subject does not feel s/he has direct and voluntary control and which occurs in the awake state" [2]. Auditory verbal hallucinations (AVHs) are defined as perceptions of speech without actual auditory stimulation and represent one of the most common hallucinatory experience, which are a characteristic symptom of schizophrenia [3] but also occur in a wealth of conditions, both neurological/neurodegenerative (e.g., epilepsy, Alzheimer's disease, Parkinson's disease, dementia, and hearing impairment) or psychiatric (e.g., dissociative disorders, mood disorders, and borderline personality disorder).

\section{TMS and Auditory Hallucinations}

Hoffman and colleagues [4] published the first report demonstrating a reduction in auditory verbal hallucinations (AVHs) after a low-frequency $(1 \mathrm{~Hz})$ rTMS treatment to the posterior superior temporal gyrus (STG). This brain area and, overall, the temporoparietal cortex (TPC) are involved in speech perception $[5,6]$ and are active during auditory hallucinations [7-9]. After the publication by Hoffman and colleagues, a number of studies replicated the positive findings using 
TABLE 1: Previous rTMS for AVHs studies.

\begin{tabular}{|c|c|c|c|c|c|c|}
\hline Study & $N$ active & $N$ sham & Stimulated Area & Frequency & $\% \mathrm{MT}$ & Session \\
\hline Hoffman et al. [44] & 12 & 12 & T3P3 & $1 \mathrm{~Hz}$ & 80 & 4 \\
\hline McIntosh et al. [45] & 16 & 16 & T3P3 & $1 \mathrm{~Hz}$ & 80 & 4 \\
\hline Schönfeldt-Lecuona et al. [46] & 11 & 10 & STG & $1 \mathrm{~Hz}$ & 90 & 5 \\
\hline Chibbaro et al. [47] & 8 & 8 & T3P3 & $1 \mathrm{~Hz}$ & 90 & 4 \\
\hline Fitzgerald et al. [48] & 17 & 15 & T3P3 & $1 \mathrm{~Hz}$ & 90 & 10 \\
\hline Hoffman et al. [49] & 27 & 23 & T3P3 & $1 \mathrm{~Hz}$ & 90 & 10 \\
\hline Lee et al. [50] & 13 & 14 & T3P3 & $1 \mathrm{~Hz}$ & 100 & 10 \\
\hline Lee et al. [50] & 12 & 14 & $\mathrm{~T} 4 \mathrm{P} 4$ & $1 \mathrm{~Hz}$ & 100 & 10 \\
\hline Poulet et al. [51] & 10 & 10 & T3P3 & $1 \mathrm{~Hz}$ & 90 & 5 \\
\hline Brunelin et al. [52] & 14 & 10 & T3P3 & $1 \mathrm{~Hz}$ & 90 & 10 \\
\hline Jandl et al. [53] & 16 & 16 & Т3Р3 Т4P4 & $1 \mathrm{~Hz}$ & 100 & 5 \\
\hline Jandl et al. [53] & 16 & 16 & $\mathrm{~T} 4 \mathrm{P} 4$ & $1 \mathrm{~Hz}$ & 100 & 5 \\
\hline Rosa et al. [54] & 6 & 5 & T3P3 & $1 \mathrm{~Hz}$ & 90 & 10 \\
\hline Saba et al. [55] & 8 & 8 & T3P3 & $1 \mathrm{~Hz}$ & 80 & 10 \\
\hline Vercammen et al. [56] & 16 & 16 & T3P3 & $1 \mathrm{~Hz}$ & 90 & 12 \\
\hline Vercammen et al. [56] & 14 & 16 & T3P3 T4P4 & $1 \mathrm{~Hz}$ & 90 & 12 \\
\hline Loo et al. [57] & 18 & 18 & T3P3 & $1 \mathrm{~Hz}$ & 110 & 3 \\
\hline Loo et al. [57] & 18 & 18 & $\mathrm{~T} 4 \mathrm{P} 4$ & $1 \mathrm{~Hz}$ & 110 & 3 \\
\hline Slotema et al. [58] & 22 & 20 & T3P3 & $1 \mathrm{~Hz}$ & 90 & 15 \\
\hline Slotema et al. [59] & 20 & 20 & fMRI & $1 \mathrm{~Hz}$ & 90 & 15 \\
\hline
\end{tabular}

MT: motor threshold; T3P3: left temporoparietal cortex; T4P4: right temporoparietal cortex; STG: superior temporal gyrus; fMRI: functional magnetic resonance imaging.

this rTMS protocol (for a complete review see Table 1), and, additionally, several meta-analyses supported the usefulness of rTMS in the treatment of hallucinations [10-12]. Most studies examined AVHs in schizophrenia, while there's a lack of studies about TMS to treat hallucinations in dementia, even though a number of studies reported the presence of psychotic symptoms (mostly delusions and hallucinations) in Alzheimer's and Parkinson's diseases (for a more detailed review see $[13,14])$.

\section{RDOCs and Circuitries Involved}

Though the exact pathophysiological mechanisms of AVHs remain actually unclear, neuroimaging studies have shown abnormalities in a distributed network of brain regions, providing valuable information about brain activity either during hallucinations or at rest. Studies on AVHs mainly employ neurophysiological methods (i.e., EEG), functional magnetic resonance imaging (fMRI), and, recently, transcranial magnetic stimulation (TMS). Most EEG studies showed changes in activity in the left superior temporal gyrus [15-18], not surprisingly since the involvement of this area in auditory processing. fMRI studies contributed much to understanding brain activity in AVHs: Allen and colleagues [19] proposed a neuroanatomical model of hallucinations including brain areas involved in linguistic processing, attention, memory, and emotion. In particular regarding AVHs, they hypothesized an altered connectivity between the superior temporal, inferior frontal gyrus and anterior cingulate cortex that might cause dysfunctional activation in language processing areas. Grounding upon the contribution of Hoffman et al. [20], the authors also hypothesized an increased activation or hypercoupling of speech production centers in the inferior frontal cortex and speech perception areas in left temporoparietal cortex. These areas seem to be specifically involved in the pathophysiology of AVHs, yet the model includes other brain regions such as Broca's area, the dorsolateral prefrontal cortex (DLPFC), orbitofrontal cortex (OFC), the supplementary motor area (SMA), the dorsal and ventral anterior cingulate $(\mathrm{DaC} ; \mathrm{VaC})$, the cerebellum, and a number of subcortical regions (Figure 1).

Recent contributions are divided into "state" studies (which investigated brain areas involved during AVH experience) and "trait" studies (which investigate the tendency to hallucinate) [21]. State studies highlighted the role of areas related to inner speech production such as the bilateral inferior frontal gyrus (IFG) and the bilateral postcentral gyrus, plus the left inferior parietal lobe (IPL) that could play a role in the misattribution of the speech as not being selfgenerated. On the other hand, trait studies focused on areas associated with speech perception such as the left superior temporal gyrus (STG) and left middle temporal gyrus (MTG) and anterior cingulate cortex (ACC) that could similar to left IPL play a role in the distorted self-monitoring of speech [22]. An involvement is also shown for putamen and Wernicke's areas and its right homologue [23].

In conclusion, studies suggest that the core mechanisms of AVHs involve a complex functional loop including several brain areas interconnected, rather than a single pathway [23]. 


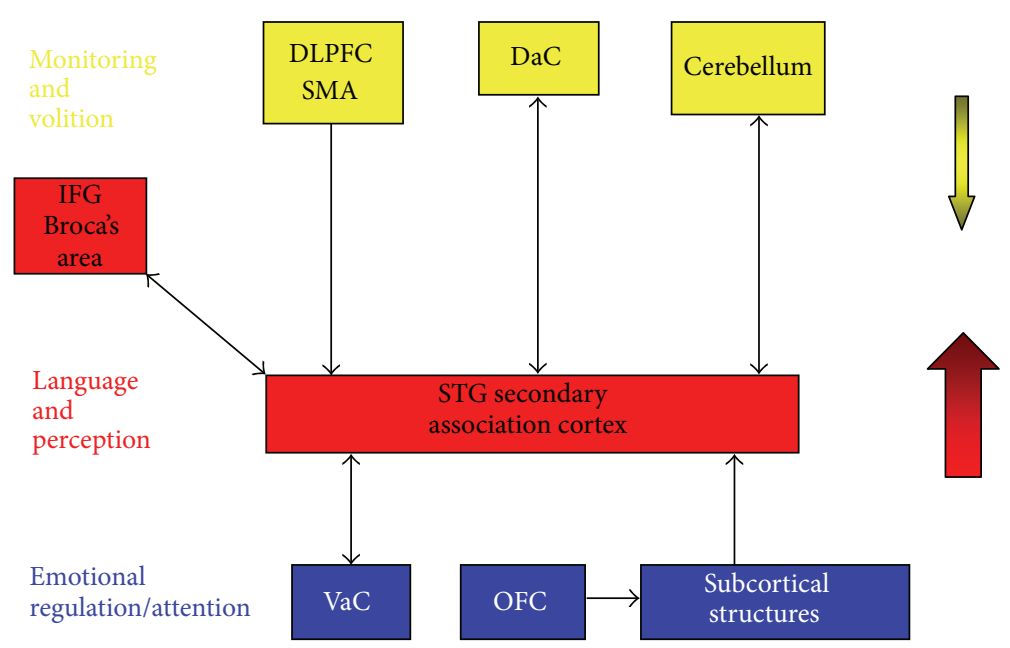

Figure 1: A neuroanatomical model of the hallucinating brain [19].

\section{Auditory Verbal Hallucinations (AVH) in Other Disorders}

Though the high prevalence in schizophrenia (40\%-80\%; [24]), AVHs are not specific for the disorder and, likewise, occur in the $10 \%-15 \%$ of nonclinical subjects $[25,26]$. In healthy subjects, the presence of AVHs is frequently associated with subclinical levels of other schizotypal phenomena, plus an increased family loading for psychosis and axis I disorders and a higher prevalence of childhood trauma, but these subjects do not meet the criteria for schizotypal, schizoid, or paranoid disorders [27]. Furthermore, a crucial difference between the emotional content of AVHs in healthy individuals and patients with schizophrenia has been observed $[28,29]$ : while in schizophrenia AVHs are characterized by a negative emotional content leading to perceived distress and to a lower global functioning, voices of the healthy subjects mainly have a positive emotional content and are often not disturbing. This difference in the emotional content could distinguish between benign hallucinations and psychopathology.

In regard to clinical conditions, AVHs are frequently found in neurological or neurodegenerative disorders such as Alzheimer's disease, Parkinson's disease, dementia, hearing impairment and epilepsy [13, 30, 31], substance abuse [32], and in psychiatric conditions such as dissociative disorders, mood disorders, and borderline personality disorder [14, $33,34]$. In particular focusing on dementia, the presenceof behavioral symptoms (mostly delusions and hallucinations) is significant, in particular in Alzheimer's disease and vascular dementia [35]: specifically, the prevalence of AVHs ranges between $1 \%$ and $29 \%$ [13]. Hallucinations seem to slightly increase correlating with the severity of dementia on the Clinical Dementia Rating [35], with greater percentage (21\%) in moderate dementia. Findings also showed that psychotic symptoms in dementia frequently lead to caregiver distress and, consequently, to institutionalization and greater cognitive deterioration $[13,14]$.

\section{Case Presentation}

A seventy-six-years-old woman presented with her daughter to the Institute, complaining of hearing disturbing voices (AVHs according to Neuropsychiatric Inventory Clinician (NPI-C; [36]) started on August 2012. She reported she firstly suffered from hypnagogic hallucinations represented by religious choirs which later turned into hearing her dead newborn son's voice. These AVHs initially had a positive content, while afterwards she began to hear threatening voices associated with sleep disturbances. On presentation, she also reported fear of the dark and being left alone at home. A partial awareness of the hallucinating origin of the voices, typical in hallucinosis, distinguished her clinical picture from AVHs in psychosis. Her insight could be assessed through the Schedule for the Assessment of Insight (SAI; [37]): the woman showed quite high insight with regard to the recognition of her abnormal mental events and actively searched for treatment, accepting therapy with good adherence.

Magnetic resonance images showed bilaterally altered areas characterizing the periventricular and subcortical supratentorial white matter, in conjunction with vascular-degenerative subcortical encephalopathy features. The woman's cognitive clinical picture was a Mild Cognitive Impairment, though the long-term duration and the clinical evidence of progression set the diagnosis of dementia.

The pharmacological treatment essentially consisted of risperidone, gradually decreased. However, AVHs has been shown to be particularly difficult-to-treat and resistant to pharmacotherapy, suggesting that the improvement reached was not attributable to pharmacological treatment.

On presentation, her cognitive function was assessed through the Mini Mental State Examination (MMSE; [38]) reaching a score of 25.24; the Symptoms Check-List 90-R (SCL 90-R; [39]) was also administered, showing high values on "obsessivity compulsivity" (40\%), "depression" (37\%), and "psychoticism" (50\%) areas. The intensity and features of the auditory verbal hallucinations were assessed during a psychiatric interview, with the aid of the Neuropsychiatric Inventory 
Clinician (NPI-C; [36]). This instrument represents a comprehensive assessment tool for neuropsychiatric symptoms and psychopathology in Alzheimer's disease and other neurodegenerative disorders. The main results showed neither delusions nor agitation, irritability, aggression, disinhibition or aberrant motor and verbal disturbances. Conversely, as previously mentioned, the patient complained about daily threatening voices which caused her sleep disturbances and, consequently, significant distress.

The woman was submitted to a $10 \mathrm{~Hz}$ rTMS treatment over T3P3 (temporoparietal cortex) at 80\% motor threshold. The protocol of administration consisted in a 15-day daily treatment, with a stimulation sequence of 13 trains of 200 stimuli each (50 seconds intertrain interval), for an amount of 2600 stimuli. After treatment, the patient reported a marked reduction in AVHs: their frequency drastically decreased, their content loss threatening features, and, consequently, the associated distress considerably lowered. Also, no side effects were reported during all treatment and in the aftermath.

\section{Discussion}

Previous studies regarding the use of TMS in the treatment of AVHs mostly employed the low-frequency rTMS protocol firstly proposed by Hoffman and colleagues [4]. Yet, to date, most literature concerning the employ of TMS in AVHs focused for the most part on schizophrenic patients, which have considerably different traits compared to elderly people with neurodegenerative disorders. In addition, the absence of delusional features in hallucinations set the patient's clinical picture markedly distant from psychosis: AVHs were not embedded in a delusional frame and not related to any particular delusional belief. Moreover, a partial awareness was preserved, almost giving the woman's AVHs the features of hallucinosis. This, in addition to neurodegenerative phenomena highlighted by MRI, explains our choice for a high frequency rTMS protocol. Given the evidence of links interruptions between strategic temporal and frontal brain regions [40], we hypothesized different features of AVHs between schizophrenia and dementia. In particular, we supposed the primary role of a disconnective syndrome rather than cortical hyperactivity [41-43] in dementia: this explains our choice of the rTMS protocol mentioned above.

On the other hand, as far as we know, the literature concerning AVHs in elderly people did not contemplate the employ of TMS as a therapeutic tool. Hence, this contribution sets in a promising field of extending the therapeutic purposes of TMS: this latter proves as potentially useful also in some difficult-to-treat neurodegenerative disorders symptoms, such as behavioral symptoms. Currently, no specific therapeutic guidelines are available for behavioral symptoms, in particular for pharmacoresistant AVHs: TMS could represent a valuable treatment because of its effect on multiple symptoms. In particular, AVHs improvement is associated with mood and cognitive improvement: we actually cannot establish a causative connection affirming which is the primary target of rTMS treatment, but, as far as we know, we can observe its effects both on AVHs, mood, and cognition. Though further research is needed, this represents a first, encouraging result: next step could be an analysis of changes in functional and metabolic brain activity after rTMS treatment.

\section{Conflict of Interests}

The authors declare that there is no conflict of interests regarding the publication of this paper.

\section{References}

[1] A. T. Barker, R. Jalinous, and I. L. Freeston, "Non-invasive magnetic stimulation of human motor cortex," The Lancet, vol. 1, no. 8437, pp. 1106-1107, 1985.

[2] A. S. David, "The cognitive neuropsychiatry of auditory verbal hallucinations: an overview," Cognitive Neuropsychiatry, vol. 9, no. 1-2, pp. 107-123, 2004.

[3] N. Sartorius, A. Jablensky, and A. Korten, "Early manifestations and first-contact incidence of schizophrenia in different cultures. A preliminary report on the initial evaluation phase of the WHO Collaborative Study on determinants of outcome of severe mental disorders," Psychological Medicine, vol. 16, no. 4, pp. 909-928, 1986.

[4] R. E. Hoffman, N. N. Boutros, R. M. Berman et al., "Transcranial magnetic stimulation of left temporoparietal cortex in three patients reporting hallucinated 'voices"' Biological Psychiatry, vol. 46, no. 1, pp. 130-132, 1999.

[5] J. A. Fiez, M. E. Raichle, D. A. Balota, P. Tallal, and S. E. Petersen, "PET activation of posterior temporal regions during auditory word presentation and verb generation," Cereb Cortex, vol. 6, no. 1, pp. 1-10, 1995.

[6] R. R. Benson, D. H. Whalen, M. Richardson et al., "Parametrically dissociating speech and nonspeech perception in the brain using fMRI," Brain and Language, vol. 78, no. 3, pp. 364-396, 2001.

[7] D. A. Silbersweig, E. Stern, C. Frith et al., "A functional neuroanatomy of hallucinations in schizophrenia," Nature, vol. 378, no. 6553, pp. 176-179, 1995.

[8] B. R. Lennox, S. B. G. Park, I. Medley, P. G. Morris, and P. B. Jones, "The functional anatomy of auditory hallucinations in schizophrenia," Psychiatry Research, vol. 100, no. 1, pp. 13-20, 2000.

[9] S. S. Shergill, M. J. Brammer, S. C. R. Williams, R. M. Murray, and P. K. McGuire, "Mapping auditory hallucinations in schizophrenia using functional magnetic resonance imaging," Archives of General Psychiatry, vol. 57, no. 11, pp. 1033-1038, 2000.

[10] A. Aleman, I. E. Sommer, and R. S. Kahn, "Efficacy of slow repetitive transcranial magnetic stimulation in the treatment of resistant auditory hallucinations in schizophrenia: a metaanalysis," Journal of Clinical Psychiatry, vol. 68, no. 3, pp. 416421, 2007.

[11] C. Freitas, F. Fregni, and A. Pascual-Leone, "Meta-analysis of the effects of repetitive transcranial magnetic stimulation (rTMS) on negative and positive symptoms in schizophrenia," Schizophrenia Research, vol. 108, no. 1-3, pp. 11-24, 2009.

[12] C. W. Slotema, J. D. Blom, H. W. Hoek, and I. E. C. Sommer, "Should we expand the toolbox of psychiatric treatment methods to include repetitive transcranial magnetic stimulation (rTMS)? A meta-analysis of the efficacy of rTMS in psychiatric 
disorders," Journal of Clinical Psychiatry, vol. 71, no. 7, pp. 873$884,2010$.

[13] M. M. Bassiony and C. G. Lyketsos, "Delusions and hallucinations in Alzheimer's disease: review of the brain decade," Psychosomatics, vol. 44, no. 5, pp. 388-401, 2003.

[14] F. Larøi, I. E. Sommer, J. D. Blom, C. Fernyhough, K. Hugdahl, L. C. Johns et al., "The characteristic features of auditory verbal hallucinations in clinical and nonclinical groups: state-of-theart overview and future directions," Schizophrenia Bulletin, vol. 38, no. 4, pp. 724-733, 2012.

[15] R. Ishii, K. Shinosaki, Y. Ikejiri et al., "Theta rhythm increases in left superior temporal cortex during auditory hallucinations in schizophrenia: a case report," Neuroreport, vol. 11, no. 14, pp. 3283-3287, 2000.

[16] U. Reulbach, S. Bleich, C. Maihöfner, J. Kornhuber, and W. Sperling, "Specific and unspecific auditory hallucinations in patients with schizophrenia: a magnetoencephalographic study," Neuropsychobiology, vol. 55, no. 2, pp. 89-95, 2007.

[17] A. Ropohl, W. Sperling, S. Elstner et al., "Cortical activity associated with auditory hallucinations," Neuroreport, vol. 15, no. 3, pp. 523-526, 2004.

[18] A. Sritharan, P. Line, A. Sergejew, R. Silberstein, G. Egan, and D. Copolov, "EEG coherence measures during auditory hallucinations in schizophrenia," Psychiatry Research, vol. 136, no. 2-3, pp. 189-200, 2005.

[19] P. Allen, F. Larøi, P. K. McGuire, and A. Aleman, "The hallucinating brain: a review of structural and functional neuroimaging studies of hallucinations," Neuroscience and Biobehavioral Reviews, vol. 32, no. 1, pp. 175-191, 2008.

[20] R. E. Hoffman, M. Hampson, K. Wu et al., "Probing the pathophysiology of auditory/verbal hallucinations by combining functional magnetic resonance imaging and transcranial magnetic stimulation," Cerebral Cortex, vol. 17, no. 11, pp. 27332743, 2007.

[21] R. van Lutterveld, Auditory verbal hallucinations and the brain [Ph.D. thesis], Rudolph Magnus Institute of Neuroscience, University Medical Center, Utrecht, The Netherlands, 2012.

[22] S. Kuhn and J. Gallinat, "Quantitative meta-analysis on state and trait aspects of auditory verbal hallucinations in schizophrenia," Schizophrenia Bulletin, vol. 38, no. 4, pp. 779-786, 2011.

[23] R. E. Hoffman, T. Fernandez, B. Pittman, and M. Hampson, "Elevated functional connectivity along a corticostriatal loop and the mechanism of auditory/verbal hallucinations in patients with schizophrenia," Biological Psychiatry, vol. 69, no. 5, pp. 407-414, 2011.

[24] A. Aleman and F. Larøi, Hallucinations: The Science of Idiosyncratic Perception, American Psychological Association, Washington, DC, USA, 2008.

[25] A. Y. Tien, "Distributions of hallucinations in the population," Social Psychiatry and Psychiatric Epidemiology, vol. 26, no. 6, pp. 287-292, 1991.

[26] V. Beavan, J. Read, and C. Cartwright, "The prevalence of voicehearers in the general population: a literature review," Journal of Mental Health, vol. 20, no. 3, pp. 281-292, 2011.

[27] I. E. Sommer, K. Daalman, T. Rietkerk et al., "Healthy individuals with auditory verbal hallucinations; who are they? Psychiatric assessments of a selected sample of 103 subjects," Schizophrenia Bulletin, vol. 36, no. 3, pp. 633-641, 2010.

[28] A. Honig, M. A. J. Romme, B. J. Ensink, S. D. M. A. C. Escher, M. H. A. Pennings, and M. W. Devries, "Auditory hallucinations: a comparison between patients and nonpatients," Journal of Nervous and Mental Disease, vol. 186, no. 10, pp. 646-651, 1998.
[29] C. Choong, M. D. Hunter, and P. W. R. Woodruff, "Auditory hallucinations in those populations that do not suffer from schizophrenia," Current Psychiatry Reports, vol. 9, no. 3, pp. 206-212, 2007.

[30] H. Matsui, K. Nishinaka, T. Miyoshi et al., "Thalamic hyperperfusion in verbal hallucination of Parkinsonian patients," Internal Medicine, vol. 46, no. 21, pp. 1765-1769, 2007.

[31] I. E. Sommer, S. Koops, and J. D. Blom, "Comparison of auditory hallucinations across different disorders and syndromes," Neuropsychiatry, vol. 2, no. 1, pp. 1-12, 2012.

[32] J. Perälä, K. Kuoppasalmi, S. Pirkola et al., "Alcohol-induced psychotic disorder and delirium in the general population," British Journal of Psychiatry, vol. 197, no. 3, pp. 200-206, 2010.

[33] C. Baethge, R. J. Baldessarini, K. Freudenthal, A. Streeruwitz, M. Bauer, and T. Bschor, "Hallucinations in bipolar disorder: characteristics and comparison to unipolar depression and schizophrenia," Bipolar Disorders, vol. 7, no. 2, pp. 136-145, 2005.

[34] D. G. Kingdon, K. Ashcroft, B. Bhandari et al., "Schizophrenia and borderline personality disorder: similarities and differences in the experience of auditory hallucinations, paranoia, and childhood trauma," Journal of Nervous and Mental Disease, vol. 198, no. 6, pp. 399-403, 2010.

[35] C. G. Lyketsos, M. Steinberg, J. T. Tschanz, M. C. Norton, D. C. Steffens, and J. C. S. Breitner, "Mental and behavioral disturbances in dementia: findings from the Cache County study on memory in aging," American Journal of Psychiatry, vol. 157, no. 5, pp. 708-714, 2000.

[36] J. L. Cummings, M. Mega, K. Gray, S. Rosenberg-Thompson, D. A. Carusi, and J. Gornbein, "The neuropsychiatric inventory: comprehensive assessment of psychopathology in dementia," Neurology, vol. 44, no. 12, pp. 2308-2314, 1994.

[37] A. S. David, "Insight and psychosis," British Journal of Psychiatry, vol. 156, pp. 798-808, 1990.

[38] M. F. Folstein, S. E. Folstein, and P. R. McHugh, "'Mini mental state. A practical method for grading the cognitive state of patients for the clinician," Journal of Psychiatric Research, vol. 12, no. 3, pp. 189-198, 1975.

[39] L. R. Derogatis, SCL-90. Administration, Scoring and Procedures Manual-I for the R, (Revised) Version and Other Instruments of the Psychopathology Rating Scales Series, School of Medicine, Johns Hopkins University, Chicago, Ill, USA, 1977.

[40] M. E. Lanna, C. E. Alves, F. K. Sudo et al., "Cognitive disconnective syndrome by single strategic stroke in vascular dementia," Journal of the Neurological Sciences, vol. 322, no. 1-2, pp. 176-183, 2012.

[41] L. C. Silbert, C. Nelson, S. Holman et al., "Cortical excitability and age-related volumetric MRI changes," Clinical Neurophysiology, vol. 117, no. 5, pp. 1029-1036, 2006.

[42] A. Alberici, C. Bonato, M. Calabria et al., "The contribution of TMS to frontotemporal dementia variants," Acta Neurologica Scandinavica, vol. 118, no. 4, pp. 275-280, 2008.

[43] G. Pennisi, R. Ferri, G. Lanza et al., "Transcranial magnetic stimulation in Alzheimer's disease: a neurophysiological marker of cortical hyperexcitability," Journal of Neural Transmission, vol. 118, no. 4, pp. 587-598, 2011.

[44] R. E. Hoffman, N. N. Boutros, S. Hu, R. M. Berman, J. H. Krystal, and D. S. Charney, "Transcranial magnetic stimulation and auditory hallucinations in schizophrenia," The Lancet, vol. 355, no. 9209, pp. 1073-1075, 2000.

[45] A. M. McIntosh, D. Semple, K. Tasker et al., "Transcranial magnetic stimulation for auditory hallucinations in schizophrenia," Psychiatry Research, vol. 127, no. 1-2, pp. 9-17, 2004. 
[46] C. Schönfeldt-Lecuona, G. Grön, H. Walter et al., "Stereotaxic rTMS for the treatment of auditory hallucinations in schizophrenia," Neuroreport, vol. 15, no. 10, pp. 1669-1673, 2004.

[47] G. Chibbaro, M. Daniele, G. Alagona et al., "Repetitive transcranial magnetic stimulation in schizophrenic patients reporting auditory hallucinations," Neuroscience Letters, vol. 383, no. 1-2, pp. 54-57, 2005.

[48] P. B. Fitzgerald, J. Benitez, J. Z. Daskalakis et al., "A double-blind sham-controlled trial of repetitive transcranial magnetic stimulation in the treatment of refractory auditory hallucinations," Journal of Clinical Psychopharmacology, vol. 25, no. 4, pp. 358362, 2005.

[49] R. E. Hoffman, R. Gueorguieva, K. A. Hawkins et al., “Temporoparietal transcranial magnetic stimulation for auditory hallucinations: safety, efficacy and moderators in a fifty patient sample," Biological Psychiatry, vol. 58, no. 2, pp. 97-104, 2005.

[50] S.-H. Lee, W. Kim, Y.-C. Chung et al., "A double blind study showing that two weeks of daily repetitive TMS over the left or right temporoparietal cortex reduces symptoms in patients with schizophrenia who are having treatment-refractory auditory hallucinations," Neuroscience Letters, vol. 376, no. 3, pp. 177-181, 2005.

[51] E. Poulet, J. Brunelin, B. Bediou et al., "Slow transcranial magnetic stimulation can rapidly reduce resistant auditory hallucinations in schizophrenia," Biological Psychiatry, vol. 57, no. 2, pp. 188-191, 2005.

[52] J. Brunelin, E. Poulet, B. Bediou et al., "Low frequency repetitive transcranial magnetic stimulation improves source monitoring deficit in hallucinating patients with schizophrenia," Schizophrenia Research, vol. 81, no. 1, pp. 41-45, 2006.

[53] M. Jandl, J. Steyer, M. Weber et al., "Treating auditory hallucinations by transcranial magnetic stimulation: a randomized controlled cross-over trial," Neuropsychobiology, vol. 53, no. 2, pp. 63-69, 2006.

[54] M. O. Rosa, W. F. Gattaz, M. A. Rosa et al., "Effects of repetitive transcranial magnetic stimulation on auditory hallucinations refractory to clozapine," Journal of Clinical Psychiatry, vol. 68, no. 10, pp. 1528-1532, 2007.

[55] G. Saba, C. M. Verdon, K. Kalalou et al., "Transcranial magnetic stimulation in the treatment of schizophrenic symptoms: a double blind sham controlled study," Journal of Psychiatric Research, vol. 40, no. 2, pp. 147-152, 2006.

[56] A. Vercammen, H. Knegtering, R. Bruggeman et al., "Effects of bilateral repetitive transcranial magnetic stimulation on treatment resistant auditory-verbal hallucinations in schizophrenia: a randomized controlled trial," Schizophrenia Research, vol. 114, no. 1-3, pp. 172-179, 2009.

[57] C. K. Loo, K. Sainsbury, P. Mitchell, D. Hadzi-Pavlovic, and P. S. Sachdev, "A sham-controlled trial of left and right temporal rTMS for the treatment of auditory hallucinations," Psychological Medicine, vol. 40, no. 4, pp. 541-546, 2010.

[58] C. W. Slotema, J. D. Blom, A. D. de Weijer et al., "Can lowfrequency repetitive transcranial magnetic stimulation really relieve medication-resistant auditory verbal hallucinations? Negative results from a large randomized controlled trial," Biological Psychiatry, vol. 69, no. 5, pp. 450-456, 2011.

[59] C. W. Slotema, J. D. Blom, H. W. Hoek, and I. E. C. Sommer, "Should we expand the toolbox of psychiatric treatment methods to include repetitive transcranial magnetic stimulation (rTMS)? A meta-analysis of the efficacy of rTMS in psychiatric disorders," Journal of Clinical Psychiatry, vol. 71, no. 7, pp. 873$884,2010$. 


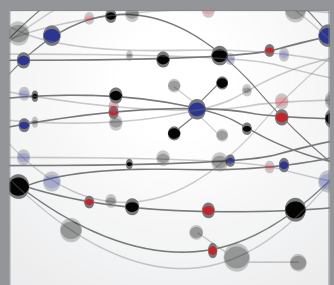

The Scientific World Journal
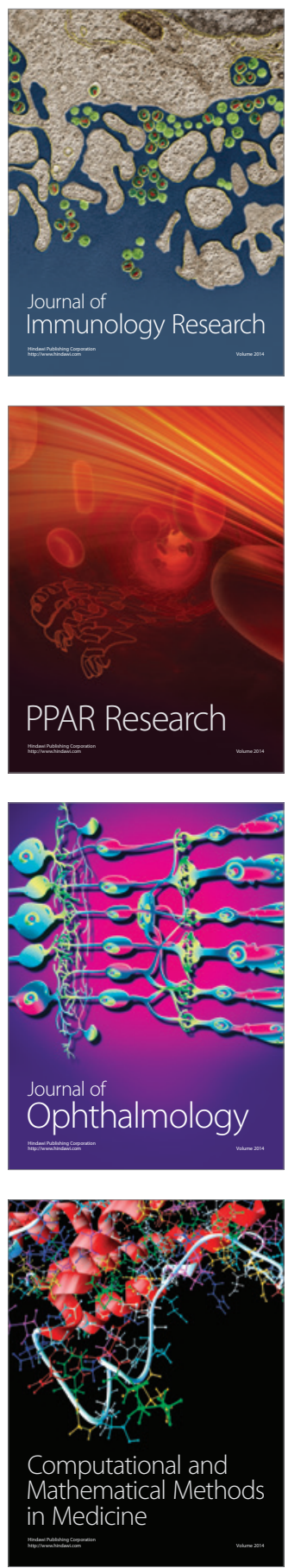

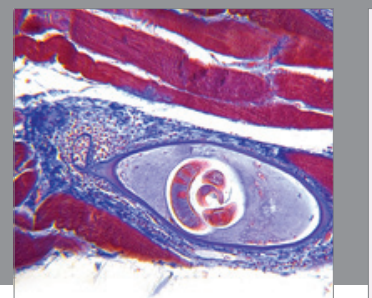

Gastroenterology

Research and Practice
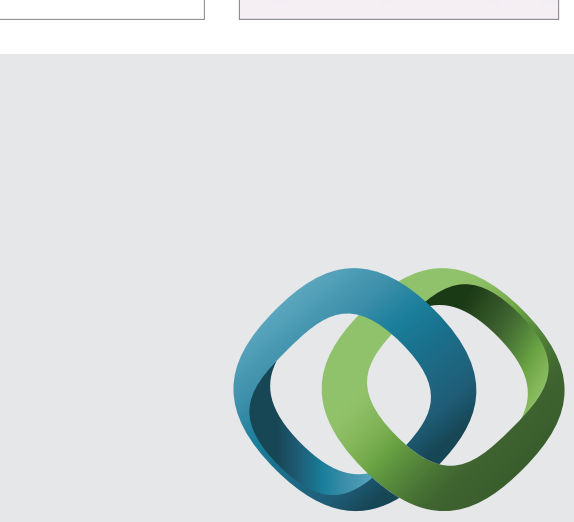

\section{Hindawi}

Submit your manuscripts at

http://www.hindawi.com
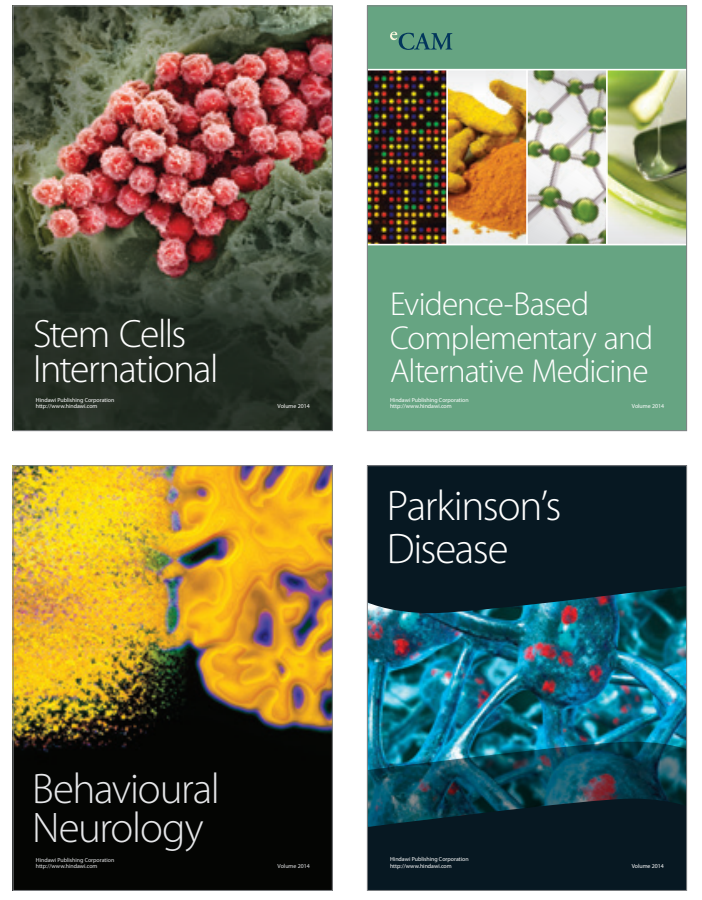
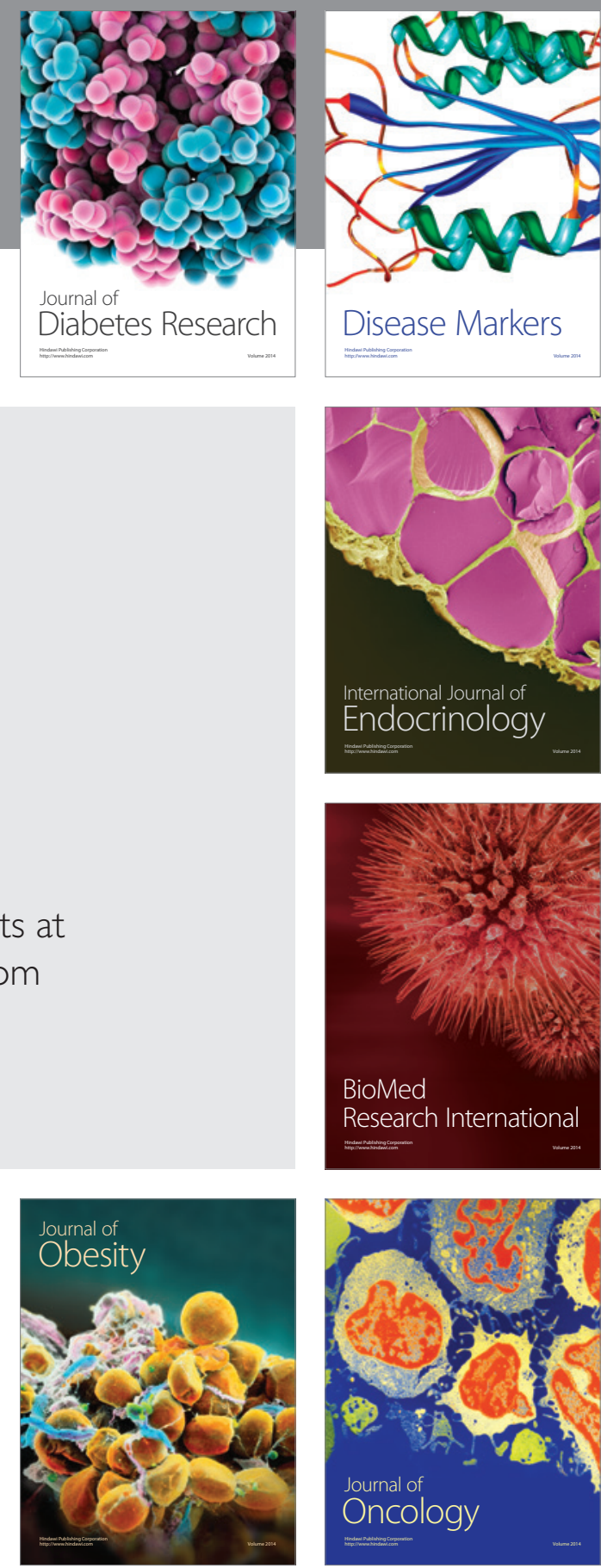

Disease Markers
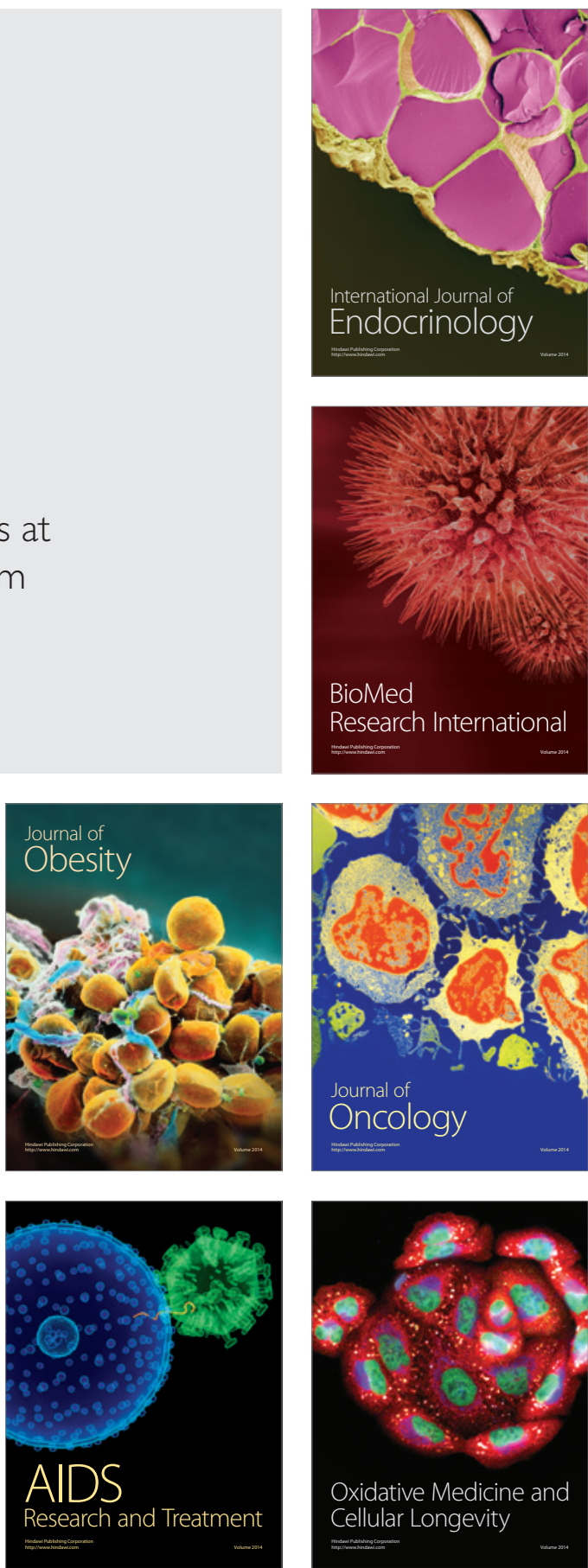